\title{
Model-Based Deformable Registration of Preoperative 3D to Intraoperative Low- Resolution 3D and 2D Sequences of MR Images
}

\author{
Bahram Marami, Shahin Sirouspour, and David W. Capson \\ Department of Electrical and Computer Engineering, McMaster University, \\ 1280 Main Street West, Hamilton, ON, Canada, L8S-4K1 \\ \{maramib, capson\}@mcmaster.ca, sirouspour@ece.mcmaster.ca
}

\begin{abstract}
We have developed an automatic model-based deformable registration method applicable to MR soft-tissue imaging. The registration algorithm uses a dynamic finite element (FE) continuum mechanics model of the tissue deformation to register its $3 \mathrm{D}$ preoperative images with intraoperative 1) 3D low-resolution or 2) 2D MR images. The registration is achieved through a filtering process that combines information from the deformation model and observation errors based on correlation ratio, mutual information or sum of square differences between images. Experimental results with a breast phantom show that the proposed method converges in few iterations in the presence of very large deformations, similar to those typically observed in breast biopsy applications.
\end{abstract}

Keywords: Image registration, soft-tissue deformation, MR imaging, breast biopsy, continuum mechanics, finite element method.

\section{Introduction}

Image registration is the process of aligning a pair of images to establish special correspondence between their features. Co-registering images obtained through multiple modalities or over a period of time can be instrumental in the diagnosis of diseases. Modern computed tomography (CT) and magnetic resonance imaging (MRI) systems can precisely image individual anatomy of human organs. However, plans based on preoperative images are not always effective during surgery because of possible movement and deformation of underlying tissue. In the last two decades, a considerable amount of effort has been devoted to research on developing intraoperative imaging techniques 1. Although interventional MRI (iMRI) systems provide intraoperative images, their long acquisition time restricts the number of $2 \mathrm{D}$ slices that can be taken during the operation. A single or even a few slices of data provide much less information than a volume of high-resolution data. Furthermore, iMRI images often have lower signal-to-noise ratio than diagnostic MR images [1].

Finite element method (FEM) discretization of the continuum mechanics based model using elastic body deformation is the most popular physical modelbased analysis in various medical applications. This method is more accurate

G. Fichtinger, A. Martel, and T. Peters (Eds.): MICCAI 2011, Part I, LNCS 6891, pp. 460-467, 2011. (C) Springer-Verlag Berlin Heidelberg 2011 
and reliable than other simpler methods such as mass-spring modeling [2]. FEMbased deformation analysis has been extensively studied for surgical simulation especially for modeling of brain shift and deformation in neurosurgery [3]. In breast imaging, FE models have been used to predict mechanical deformations during MRI-guided biopsy [4], and to validate non-rigid registration algorithms. Moreover, they have been explored to model multi-object deformations in abdominal region for deformable image registration 5. Similarities (or differences) between two sets of image data to be matched are used to deform the FE model utilized in non-rigid registration. In most cases, local information in images such as objects' surfaces and extracted feature points are employed to find external and internal forces applied to a discretized mesh [3. In [4, landmarks are tapped to the breast surface in order to track its movement during biopsy. One main drawback of these methods is that they require user intervention. Another shortcoming is that surface construction via edge detection and feature extraction is a difficult and unreliable process especially in multi-modal image matching.

In this paper we propose a FE model based deformable registration method to volumetrically register high-resolution undeformed MR images of a breast phantom to low-resolution deformed data. We also provide an algorithm to nonrigidly register $3 \mathrm{D}$ undeformed images to $2 \mathrm{D}$ slices of the deformed data. In the proposed method a dynamic FE model acts as a regularization constraint on the image similarity criterion. The model not only leads the nonlinear optimization problem involved in registration process to its global extremum, but also provides temporal correlation for information obtained through $2 \mathrm{D}$ slices over time. This would allow tracking of the $3 \mathrm{D}$ volume deformation of soft-tissue based on intraoperative 2D sequences of MR images using a filtering process. In the volumetric registration, a voxel intensities based variational method is used to implicitly compute the forces applied to the 3D model. The same method is employed to find displacements of the regular $2 \mathrm{D}$ grid of the preoperative volume to match it with the $2 \mathrm{D}$ intraoperative image.

\section{Methods}

\subsection{Mathematical Formulation of the Registration Problem}

Given a reference image $R$ and a template image $T$, image registration is basically finding a reasonable displacement field $u$, so that the transformed image $T[u]$ is similar to $R$. The objective function to be minimized is:

$$
J(u)=I(T[u], R)+\alpha S(u) ; \quad \alpha \in \Re_{+}
$$

where $I$ is the distance (similarity) measure between two images. In this paper, three different measures are considered: sum of squared differences (SSD) between the images which is a distance measure, mutual information (MI) and correlation ratio $(\mathrm{CR})$ of two image data which are both similarity measures. In (1), $S$ is a regularization term which ensures that the displacement field is "reasonable". The linear elastic energy of the deformable body is used as the 
regularization term in our work 3445 . Finally $\alpha$ weighs the importance of the regulation term compared with the distance (similarity) measure.

In a linear elastic continuum with no initial strains or stresses the potential energy of a body subjected to externally applied forces can be expressed as [6]

$$
E=\int_{\Omega} \sigma^{T} \epsilon d \Omega+\int_{\Omega} u^{T} f d \Omega
$$

where $f$ is the vector of forces applied to the elastic body, $u$ the displacement field, and $\Omega$ is the body of the elastic subject. Also, $\epsilon$ and $\sigma$ are the strain and stress vectors respectively which are explained in details in 677. Based on the concept of a FE discretization, a volume of elastic body is approximated as an assemblage of discrete finite elements interconnected at nodal points on the element boundaries. Hence, displacements within the elastic body can be written as a function of the displacements at the element's nodal points weighted by the element's shape function [7. Discretizing the continuous body with tetrahedral elements, for the volume of every tetrahedral element $e l$ with 4 vertices points, the potential energy function at every node $i$ (2) can be written as:

$$
E\left(u_{i}^{e l}\right)=\int_{\Omega} \sum_{j=1}^{4} u_{i}^{e l^{T}} B_{i}^{e l^{T}} D B_{j}^{e l} u_{j}^{e l} d \Omega+\int_{\Omega} u_{i}^{e l^{T}} f_{i}^{e l} d \Omega
$$

Here $u_{i}^{e l}$ is the nodal points displacement, $f_{i}^{e l}$ is the vector of forces concentrated at the nodal points, $D$ is the elasticity matrix characterizing the material's property, and $B_{i}^{e l}$ depends on the shape function [6]. $f_{i}^{e l}$ can be computed as a classical optical flow field between the images to be matched; this would provide a semi-implicit method where the optical flow field would be an initial estimate of the deformation field which is regularized by the elastic model [7]. In the discretized domain, the objective function to be minimized (1) would become:

$$
J\left(u_{i}^{e l}\right)=I\left(T\left[u_{i}^{e l}\right], R\right)+\alpha \int_{\Omega} \sum_{j=1}^{4} u_{i}^{e l^{T}} B_{i}^{e l}{ }^{T} D B_{j}^{e l} u_{j}^{e l} d \Omega
$$

If $J$ has a local extremum at $u_{i}^{e l}$, its first variation at $u_{i}^{e l}$ must vanish, i.e. $\frac{d J\left(u_{i}^{e l}\right)}{d u_{i}^{e l}}=0$. Then using Eq. (4), we have:

$$
\frac{d I\left(T\left[u_{i}^{e l}\right], R\right)}{d u_{i}^{e l}}+\alpha \int_{\Omega} \sum_{j=1}^{4} B_{i}^{e l}{ }^{T} D B_{j}^{e l} u_{j}^{e l} d \Omega=0
$$

Comparing (3) and (4), and considering forces $f_{i}^{e l}$ concentrated at the nodal points, (5) can be written in a matrix form for each element as $K^{e l} u^{e l}=f^{e l}$ where $K_{i, j}^{e l}=\int_{\Omega} B_{i}^{e l}{ }^{T} D B_{j}^{e l} d \Omega$ is a $3 \times 3$ matrix and every element $i, j$ refers to pairs of nodes of the element $e l ; f_{j}^{e l}=-\frac{1}{\alpha} \frac{d I\left(T\left[u_{j}^{e l}\right], R\right)}{d u_{j}^{e l}}$ is a $3 \times 1$ vector. The 
$12 \times 12$ matrices $K^{e l}$ and the vector $f_{j}^{e l}$ are computed for each element and are then assembled in a global system as:

$$
K u=f(u)
$$

where $K$ is the global stiffness matrix associated with the volumetric mesh, $u$ is the vector of nodal displacements and $f(u)$ is the vector of nodal forces. The solution to this nonlinear system of equations will provide the displacement field corresponding to the global minimum of the objective function (1).

\subsection{Dynamic Finite Element Model}

An iterative numerical method has to be employed to solve the nonlinear system of equations in (6). To this end, in this paper we consider a second-order dynamical system of the form of [8]

$$
M \ddot{u}+C \dot{u}+K u=f(u)
$$

where $M$ is the mass matrix of the elements concentrated at nodes, and $C=$ $\beta M+\gamma K$ is the damping matrix for constant values of $\beta$ and $\gamma$. It is noted that the steady-stated equilibrium of the this dynamical system is the solution to the static system of equations in (6). The dynamic equations can be solved using existing implicit or explicit numerical integration routines over time.

This dynamic model also allows for real-time intraoperative registration of a deforming organ in our approach. It provides a temporal correlation model for the images taken at different sample times. Such situation can arise, for example, in real-time MR based biopsy interventions where the soft tissue undergoes deformation due to the force of needle insertion. In this paper a modal-based model reduction is employed to solve (7) more efficiently 8]. Using this method, very fast modes of (7) are eliminated to significantly reduce computations without affecting the steady-state solution.

\subsection{D Volumetric Registration}

In the volumetric registration, 3D high-resolution and 3D low-resolution MR images of an undeformed and deformed breast phantom are the template and reference image data sets, respectively. The nodal forces are computed based on the derivative of the distance (similarity) measure. By letting $h$ denote a generic intensity comparison function, $f(u)$ at each iteration can be computed as:

$$
f(u)=-\frac{1}{\alpha} h(T[u], R) \nabla T[u]
$$

where $\nabla T[u]$ is the gradient of the template image in every iteration. The exact form of $h(\cdot, \cdot)$ for three (similarity) measures, i.e. SSD, CR and MI, are given in 9]. The nodal forces computed from (8) are applied to the dynamic model in (7) and the resulting displacement field is used to interpolate a new 3D template image. This new template image is used to calculate the nodal forces in the next iteration. The process continues until the solution converges to an equilibrium. 


\subsection{D-3D Deformable Registration}

This section presents a method for deformable registration of a 3D preoperative (undeformed) MR image to one or more 2D intraoperative (deformed) MR images. For any 2D slice of the intraoperative image data, the corresponding $2 \mathrm{D}$ slice of the preoperative image data is found based on the position and the orientation of the virtual 2D iMRI plane. We employ a method similar to that of Section 2.3 to find the displacement of voxel points to match these two slices. Hence, for every point of the regular grid $y$, its displacement $d y=-\lambda h(T[u(y)], R) \nabla T[u(y)]$ where $\lambda \in \Re_{+}$is a constant gain. It should be noted that $d y$ is a $3 \times 1$ vector, i.e. the computed displacement is not restricted to the plane of the $2 \mathrm{D}$ slice. The shape functions of the elements relate the displacements of regular grid points of the $2 \mathrm{D}$ slice to those of the nodal points of the FE mesh in which these regular points reside. This essentially constitutes the process measurement in our filtering approach to the deformation estimation.

The discretized version of the dynamic deformation model in (7) can be written in the following general state-space form:

$$
x(k+1)=A x(k)+G f \quad z(k)=H x(k)
$$

where $x$ is the vector of system states, i.e., nodal displacements and velocities, and $z(k)$ is the vector of measurements, i.e., the computed displacements at the regular 2D grid points. Moreover, $A, G$, and $H$ are the state transition matrix, the model input matrix and the model output matrix, respectively. Having the model (9) and the measurements, one can iteratively estimate the system states using a Kalman-type filtering process [10]. The deformed 3D intraoperative image can then be obtained by an interpolation of the deformed $\mathrm{FE}$ mesh.

\section{$3 \quad$ Experiments and Results}

A triple modality biopsy training breast phantom (CIRS model 051 ) is used for obtaining the experimental data. A 3D volume high-resolution $(512 \times 512 \times 136)$ set of MR images has been taken from the undeformed phantom using a GE 3 T Signa MRI machine. Also, two sets of 3D high-resolution $(512 \times 512 \times 136)$ and low-resolution $(64 \times 64 \times 32)$ are taken from the deformed phantom. Fig. [1 shows the apparatus developed for deforming the phantom and two sample images taken in undeformed and deformed states. The device in Fig. 1(a) is made of plexiglass and is MR compatible. Four capsules of vitamin $\mathrm{E}$ are attached to the device as landmarks to rigidly register the reference coordinate frames of the deformed and undeformed image data sets. An isotropic linear elastic deformation model with the Young's elasticity modulus $E=10^{5}$ and Poisson's ratio $\nu=0.45$ has been used in the experiments. Since the deformation model merely acts a constraint in the registration process, these parameters could essentially be tuned by the user to achieve a desirable outcome. A cubic mesh of tetrahedral elements encompassing the whole volume of the deformed and undeformed data is created using the COMSOL Software. Fig. 2 depicts the volumetric mesh of 


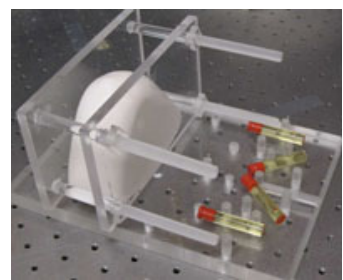

(a)

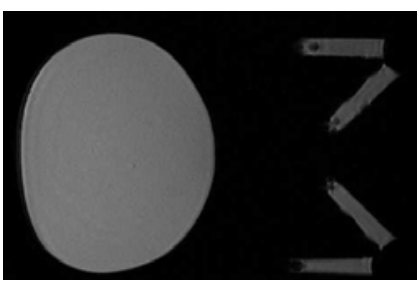

(b)

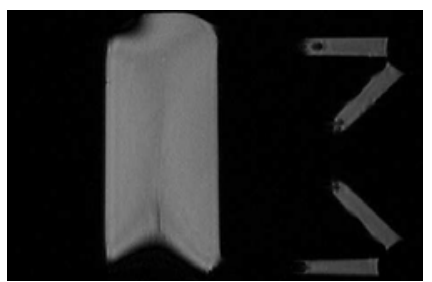

(c)

Fig. 1. The experimental setup and sample images: (a) the apparatus used for deforming the breast phantom, (b) image of undeformed phantom, (c) image of deformed phantom

7502 elements and 1601 nodal points before (a) and after (b) deformation. It is clear from this figure that the mesh has to translate and deform in order to cope with the large deformation in the phantom. It should be noted that our approach requires no segmentation of the preoperative image since it does not use a specific geometry for the mesh and applies forces to all nodes.

The evolution of three different distance (similarity) measures in the iterative registration of $3 \mathrm{D}$ high-resolution to low-resolution are shown in Fig. 2(c). For this experiment, the registration is done based on the SSD measure, and $\mathrm{CR}$ and MI are computed and used for comparison in each iteration. It is evident that the algorithm converges to a solution after about 15 iterations. It is also shown in Fig. 3 that registering the 3D high-resolution preoperative image data (a) to the 3D low-resolution intraoperative image data (b) produces a deformed preoperative image (c) which is very similar to the actual high-resolution intraoperative image (d). A quantitative comparison of the three distance (similarity) measures for three different measures based registration is given in Table 1. For a better comparison, normalized mutual information (NMI) is computed instead of $\mathrm{MI}$ because $0 \leq \mathrm{NMI} \leq 2$ for any two sets of image data. The optimal values of the image similarity metrics are zero for SSD, 1 for $\mathrm{CR}$, and 2 for NMI. In Table 1 different distance (similarity) measures for the

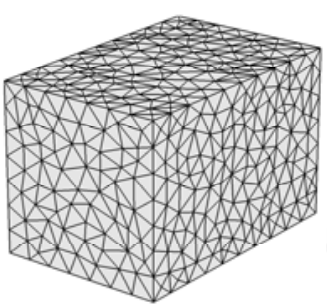

(a)

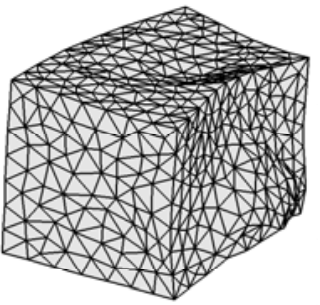

(b)

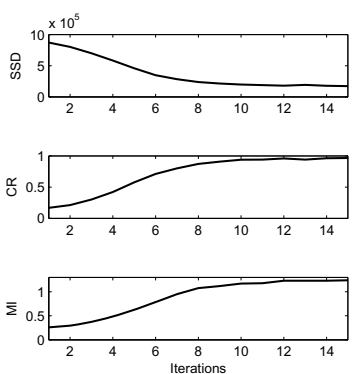

(c)

Fig. 2. (a) undeformed mesh, (b) deformed mesh, (c) distance (similarity) measures 


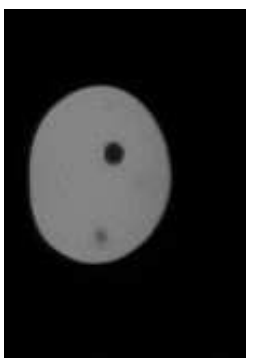

(a)

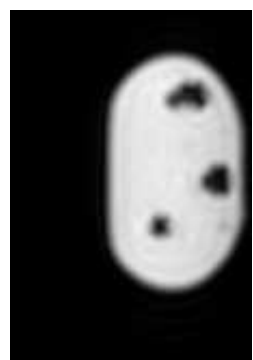

(b)

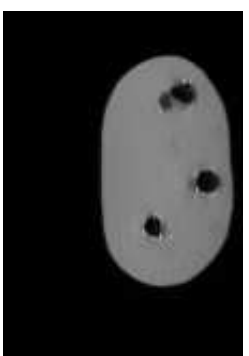

(c)

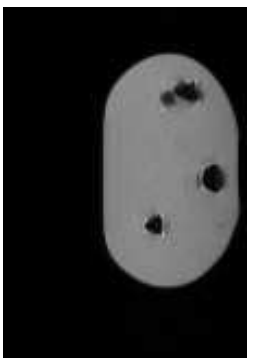

(d)

Fig. 3. The $x-y$ view of the (a) preoperative images (template data), (b) low-resolution intraoperative images (reference data), (c) deformed preoperative images after registration, (d) actual high-resolution intraoperative image

Table 1. Distance (similarity) measures for images with (0-255) gray scale values

\begin{tabular}{|l|c|c|c||c|c|c|}
\cline { 2 - 7 } \multicolumn{1}{c|}{} & \multicolumn{3}{c||}{ 3D volumetric registration } & \multicolumn{3}{c|}{ 2D-3D registration } \\
\hline Registration & SSD & CR & NMI & SSD & CR & NMI \\
\hline SSD based & $139 \times 10^{6}$ & 0.941 & 1.151 & $167 \times 10^{6}$ & 0.910 & 1.061 \\
\hline CR based & $141 \times 10^{6}$ & 0.956 & 1.159 & $172 \times 10^{6}$ & 0.918 & 1.078 \\
\hline MI based & $152 \times 10^{6}$ & 0.923 & 1.143 & $181 \times 10^{6}$ & 0.882 & 0.970 \\
\hline
\end{tabular}

case of 2D-3D registration are also given. These measures are computed between the $3 \mathrm{D}$ volume of the actual high resolution intraoperative images and the $3 \mathrm{D}$ volume of deformed preoperative images obtained through registration. In this case, 15 slices of the $3 \mathrm{D}$ volume from various orientations are used and for each slice the iterative Kalman-type filter is executed for 10 time steps.

Table 1 shows that CR-based registration yields the most optimal values of the three metrics, especially in volumetric low-high registration. One reason for the poor performance of MI-based registration may be the rather low resolution images used for registration and the highly nonlinear nature of this similarity measure. Moreover, the results for 3D volumetric registration is better than 2D$3 \mathrm{D}$ registration. The $2 \mathrm{D}-3 \mathrm{D}$ method could be improved using better estimation methods and more $2 \mathrm{D}$ slices over time.

\section{Discussion and Conclusion}

A model-based deformable image registration method has been proposed to register high-resolution 3D volume of preoperative MR images to either lowresolution $3 \mathrm{D}$ volume or $2 \mathrm{D}$ sequences of intraoperative $\mathrm{MR}$ images. Within a filtering and estimation framework, the approach employs a continuum mechanics based model of deformation and similarity (distance) measures such as SSD, $\mathrm{CR}$, and $\mathrm{MI}$ to non-rigidly register the images. The method converges in few iterations in the presence of large deformations in the experiments with a breast 
biopsy phantom. The registration algorithm is based on voxel intensities and requires no feature extraction or image segmentation.

The proposed registration method involves tasks such as three-linear interpolation, solving the equilibrium equations in dynamic FE analysis using the Newmark method [8], finding grid points deformation based on the shape function and matrix computations, which are all computationally expensive. However these tasks are highly amenable to parallel computing using graphics processing units (GPUs). We are currently working on the implementation of the algorithms on a GPU. Our preliminary results for SSD based volumetric registration indicate a factor of 60 speedup over an optimized CPU-based implementation. In future we will extend the algorithm for multi-modal registration. In particular, we will investigate 2D-3D US to MR registration for medical diagnostic as well interventional applications.

Acknowledgments. The authors would like to thank Dr. Michael Noseworthy for providing access to the MRI machine, and Dr. Jan Modersitzki and Dr. Shahram Shirani for their comments and feedback on the work.

\section{References}

1. Fahlbusch, R.: Development of Intraoperative MRI: A Personal Journey. Itraoperative Imaging 109, Part 1, 9-16 (2011)

2. Gibson, S.F.F., Mirtich, B.: A Survey of Deformable Modeling in Computer Graphics. TR-97-19 (1997), http://www.ncbi.nlm.nih.gov

3. Ferrant, M., Nabavi, A., Macq, B., Jolesz, F.A., Kikinis, R., Warfield, S.K.: Registration of 3-d intraoperative MR images of the brain using a finite-element biomechanical model. IEEE Trans. on Med. Imaging 20(12), 1384-1397 (2001)

4. Azar, F.S., Metaxas, D.N., Schnall, M.D.: A finite element model of the breast for predicting mechanical deformations during biopsy procedures. In: IEEE Work. on Math. Meth. in Biomed. Ima. Anal., pp. 38-45. Hilton Head Island, SC, USA (2000)

5. Brock, K.K., Sharpe, M.B., Dawson, L.A., Kim, S.M., Jaffray, D.A.: Accuracy of finite element model-based multi-organ deformable image registration. Med. Phys. 32(6), 1647-1659 (2005)

6. Zienkewickz, O.C., Taylor, R.L.: The Finite Element Method. McGraw Hill, New York (1987)

7. Ferrant, M., Warfield, S.K., Guttmann, C.R.G., Mulkern, R.V., Jolesz, F.A., Kikinis, R.: 3D Image Matching Using a Finite Element Based Elastic Deformation Model. In: Taylor, C., Colchester, A. (eds.) MICCAI 1999. LNCS, vol. 1679, pp. 202-209. Springer, Heidelberg (1999)

8. Bathe, K.J.: Finite Element Procedures. Prentice Hall, Englewood Cliffs (1996)

9. Chefd'Hotel, C., Hermosillo, G., Faugeras, O.: A Variational Approach to MultiModal Image Matching. In: IEEE Wsh. on Var. Le. Set Meth., Canada, pp. 21-28 (2001)

10. Welch, G., Bishop, G.: An Introduction to the Kalman Filter. Uni. of North Carolina, TR-95-041 (2006) 